\title{
Bridging the translation gap and building the translation platform: translational medicine at Peking Union Medical College Hospital
}

\author{
Jun Xu, Shaojing Liu \& Xuezhong $\mathrm{Yu}^{*}$ \\ Peking Union Medical College Hospital, Beijing 100730, China
}

Citation: $\quad \mathrm{Xu}, \mathrm{J} ., \mathrm{Liu}, \mathrm{S}$. , and Yu, X. (2016). Bridging the translation gap and building the translation platform: translational medicine at Peking Union Medical College Hospital. Sci China Life Sci 59, 1048-1050. doi: 10.1007/s11427-012-4363-2

Although there have been progressive advancements in the life sciences since the beginning of the 21 st Century, basic medical sciences generally fail to be efficiently translated into clinical research and ultimately into clinical practice. Translational medicine has become a fashionable term describing the inclination of basic science and clinical researchers to ultimately help patients through facilitating the incorporation of basic research findings into clinical practice (Zerhouni, 2005).

The development of translational medicine in recent years has been rapid - focusing on a new medical research model advocating "patient-centered" clinical work. Starting with clinical questions regarding patient problems, conducting basic research, and then turning the results of basic research quickly into clinical applications to improve clinical care is the heart of this new model. Translational medicine is an emerging area comprising multidisciplinary research from both basic and clinical medical sciences which can be summarized by both the "bench-to-bedside" and "bedside-to-bench" concepts (Albani and Prakken, 2009). This concept requires close collaboration between clinical and basic science researchers across departments and institutions. This means translational medicine is not a traditional one-way (e.g., "bench-to-bedside"), but an open, two-way path. Labora- tory research results are not only quickly applied, but clinical practice and clinical problems give timely feedback to the laboratory in order to fine-tune and continuously improve the research process. In this article, we will play a special emphasis on translational medicine at our home institution, Peking Union Medical College Hospital

*Corresponding author (email: yxzpumch@126.com)
(PUMCH).

\section{THE TRADITION OF TRANSLATIONAL MEDICINE AT PUMCH}

The concept of translational medicine is not new at PUMCH. From the beginning, PUMCH has adhered to the concept of translational medicine by closely integrating clinical and basic science research. Not only is this represented in the unity of the hospital and medical school, but also the unity of clinical practice and basic medical research which has made PUMCH and Peking Union Medical College (PUMC) the cradles of translational medicine in China.

PUMCH has a long history and a formidable tradition in translational medicine research. In the past, many professors of PUMCH were not only distinguished physicians, but were also basic science researchers in their own right. For example, Prof. Shihao Liu, a renowned endocrinologist as well as a professor of biochemistry, devoted his life's work to the establishment and development of endocrine disciplines in China. Prof. Liu recognized that endocrinology plays a very important role in both clinical medicine and basic science. His most important contribution was a study on calcium and phosphorus metabolism performed with his colleague (and fellow endocrinologist) Xianyi Zhu. Together, their studies in the 1940s on calcium and phosphorus metabolism in osteomalacia proved that osteomalacia was mainly caused by vitamin D deficiency, and also proposed the first concept of renal osteodystrophy, as well as published the "Treatment of Renal Osteodystrophy with Dihydrotachysterol (A.T.10) and Iron" in Science (Liu and Chu, 1942). 


\section{THE ADVANTAGES OF TRANSLATIONAL MEDICINE AT PUMCH}

\section{Outstanding clinical environment}

Translational Medicine should be regarded as a two-way road which aims to bridge the gap and build the platform between basic and clinical research. To build an outstanding translational medicine team, it is not enough to have a good basic science research team, but a good clinical science team with an excellent clinical environment is crucial as well.

PUMCH and its affiliated medical college were founded by the Rockefeller Foundation in 1921. The hospital has gathered notable physicians, medical professionals and scientific researchers of various specialties since, and has maintained its leading position as one of the top-ranked hospitals in China for over 90 years. For example, PUMCH currently hosts the Chinese Academy of Medical Sciences (CAMS) on its campus. On a noted list of China's best hospitals, the comprehensive clinical strength of PUMCH ranked the highest. Every year there are more than 200 million outpatient visits and over 50,000 hospitalized patients. Meanwhile, there are more than 60,000 outpatient visits in the Health Examination Center of PUMCH each year, which can be used as a normal control group for translational medical research.

Given this immense volume of clinical encounters, PUMCH collects a very large amount of tissue and serum samples (more than 40,000 surgical specimens and 60,000 blood specimens every year). PUMCH has a long history of preserving tissue samples, and some departments at PUMCH have their own tissue banks.

At the same time, it is very important to have high quality and comprehensive medical records for medical research. Since the establishment of PUMCH, all medical records have been meticulously preserved, which can provide additional clinical data for translational medical research.

\section{Clinically-oriented medical research}

The core research principle at PUMCH is very similar to the philosophy of translational medicine. Translational medicine's "patient-centered" clinical work model has been consistently advocated at PUMCH, and likely is a key reason why PUMCH has maintained its position at the highest level of medicine in China. For example, Prof. Zhinan Zhang, in a recent article about research at PUMCH, has pointed out that "we should find problems from clinical practice and ask questions, (and) do further clinical analysis or experimental research to seek answers to solve clinical problems".

\section{Solid foundation of medical research}

The development of translational medicine must depend on a solid foundation of medical research. Currently, there are 25 professionals approved to make clinical experiments by the Chinese State Food and Drug Administration at PUMCH. PUMCH also has many high-level institutes of basic medical research, such as the International Clinical Pharmacology Center, and the Key Laboratory of Endocrinology. This existing infrastructure has enabled PUMCH to actively pursue translational medicine research. Finally, PUMCH is in close cooperation with the institutes of Pathogen Biology and Basic Medical Sciences in the Chinese Academy of Medical Sciences, as well as the Institute of Materia Medica of PUMC. All of these institutions help promote the development of basic science research and translational medical research at PUMCH.

\section{THE STATUS OF TRANSLATIONAL MEDICINE AT PUMCH}

Even though PUMCH has a plethora of basic science and clinical resources, recent steps have improved adoption of translational medicine. A first step toward the promotion of translational medicine at PUMCH was to conduct joint projects with the Institute of Basic Medical Sciences of the Chinese Academy of Medical Sciences. All projects included two research arms, basic and clinical, which worked together closely. PUMCH also co-organized the "Clinical Research Centre of Pathogen(s)" with the Institute of Pathogen Biology of the Chinese Academy of Medical Sciences to look into newly emergent pathogens.

In addition, PUMCH has held multiple international forums on translational medical research. Four forums specific to translational medical research were held from 2007 to 2011. These forums built a framework for communication between clinical and basic science researchers at PUMCH and around the world. Additionally, PUMCH was a co-organizer of the "Sino-American Symposium on Clinical and Translational Research" held in Beijing in June 2010. This meeting aimed at gathering a broader audience of clinicians, basic and clinical science researchers, ethicists and health care officials from hospitals, academia and governmental agencies involved in human subject research and/or multi-national clinical trials. A number of important themes relevant to bilateral collaborations between the USA and China were discussed (Guan and Chen, 2011).

Another milestone was the initiation of the PUMCH Translational Medicine Center (PUMCH-TMC), launched in Beijing on September 16, 2010 with the support of Ministry of Health and Ministry of Science and Technology of China. The PUMCH-TMC is a translational medical research platform involving 54 academicians based in hospital and existing medical research institutions. The objective of the PUMCH-TMC is to become a national center for translational medicine, and to assist in clinical care at PUMCH (Zhao, 2011). 
The directors of the PUMCH-TMC recognized that translational medicine is not only a concept, but requires practical action. For such, the PUMCH-TMC has identified major translational medicine research topics to focus on, including: regenerative therapy using stem cells for central nervous system diseases, the integrated study of the clinical features and molecular mechanisms of pancreatic cancer, HIV/AIDS, chronic metabolic diseases such as diabetes, rheumatologic diseases, and the diagnostic and therapeutic use of allergens. Finally, the PUMCH-TMC has recently been working with the University of California in San Francisco to work on international translational medicine collaborations.

\section{THE FUTURE GOALS OF TRANSLATIONAL MEDICINE AT PUMCH}

The future of translational medicine at PUMCH has short-, mid- and long-term goals.

Short-term goals: (i) To examine ongoing clinical and basic science research projects and use the PUMCH-TCH resources to further potential translational work. (ii) To continue to enlarge the human tissue and blood bank at $\mathrm{PUMCH}$, and make it more accessible for translational research.

Mid-term goals: A translational medicine platform should be created to provide consulting services and research technique support to interested individuals and institutes. All projects initiated by this platform should be conducted within the PUMCH-TMC. The platform should have the following functions: provide consulting services and technique support to researchers, create more collaborations and partnerships, as well as provide lectures for post- graduate education.

Long-term goals: An international translational medicine institute will be created. This international translational medicine institute will become a high-end platform for promoting international translational medicine research programs.

\section{CONCLUSION}

Translational medical research aims to provide an opportunity for patients to benefit from the closer cooperation of the basic and clinical sciences. Promising collaborative translational research as illustrated in this review is an ongoing process at PUMCH. The extensive clinical and basic medical research resources of $\mathrm{PUMCH}$ provide a robust platform for translational medicine research programs. With consistent effort, the PUMCH-TMC aims to become an international platform for supporting translational medicine research.

Compliance and ethics The author(s) declare that they have no conflict of interest.

Albani, S., and Prakken, B. (2009) The advancement of translational medicine-from regional challenges to global solutions. Nat Med 9, 1006-1009.

Guan, J., and Chen, J. (2011). Translational research and its effects on medicine in China. Chin Med J 124, 3170-3175.

Liu, S.H., and Chu, H.I. (1942). Treatment of renal osteodystrophy with dihydrotachysterol (A.T.10) and iron. Science 95, 388-389.

Zhao, Y.P. (2011). Translational medicine at Peking Union Medical College (PUMCH): past, present and future. Trans Med Res (Electronic Edition) 1, 1-7.

Zerhouni, E.A. (2005). Translational and clinical science-time for a new vision. N Engl J Med 353, 1621-1623.

Open Access This article is distributed under the terms of the Creative Commons Attribution License which permits any use, distribution, and reproduction in any medium, provided the original author(s) and source are credited. 